\title{
Comparison between REBT and Visual/Kinaesthetic Dissociation in the Treatment of Panic Disorder: An Empirical Study
}

\author{
Simon D. R. Simpson • Windy Dryden
}

Published online: 21 July 2011

(C) Springer Science+Business Media, LLC 2011

\begin{abstract}
The aim of this study was to test the efficacy of two brief treatment methods for panic disorder: Rational Emotive Behaviour Therapy (REBT) and Visual/Kinaesthetic Dissociation (VKD), neither of which have been the object of scientific enquiry. The study is a two-way between-groups pre-test/post-test experimental design with baseline and follow-up measures. An innovative foursession treatment protocol was developed for each treatment method. Eighteen participants in North-East Surrey, England, who responded to media advertisements for cognitive-behavioural treatment for panic disorder and who met Diagnostic and Statistical Manual of Mental Disorders criteria for panic disorder with or without agoraphobia were randomly assigned to either REBT or VKD. Pre-test/post-test changes in panic were measured using the ACQ, PASQ, and HADS scales and a global panic rating measure. At post-test there was a statistically significant improvement on all measures for both groups, which was maintained at one-month follow-up. Taking into consideration limitations such as the small sample size and a short follow-up period, implications of this study and recommendations for future research are discussed.
\end{abstract}

Keywords Rational emotive behaviour therapy (REBT) - CBT ·

Visual/kinaesthetic dissociation (VKD) - Imagery rescripting ·

Hypnosis · Panic

\author{
S. D. R. Simpson $(\bowtie)$ \\ 18 Lower Green Road, Esher, Surrey KT10 8HD, UK \\ e-mail: info@cbt-surrey.co.uk \\ W. Dryden \\ Goldsmiths University of London, London, UK
}




\section{Introduction}

Panic disorder (PD) is a significant health problem and is one of the most common psychiatric disorders in the United Kingdom. According to Clark (1996), community surveys indicate that between 7 and $28 \%$ of the normal population will experience an occasional unexpected panic attack at some point in their life. The prevalence rates for those who go on to develop repeated panics and panic disorder have been estimated at between 2 and $5 \%$ of the general population. The lifetime prevalence of agoraphobia (usually with panic disorder) is just under $6 \%$ (Clark 1996; Beamish et al. 2002). A panic attack according to DSM-IV (American Psychiatric Association, APA 1994) criteria is defined as a sudden episode of intense fear accompanied by at least four symptoms such as breathlessness, palpitations, dizziness, nausea, a fear of dying, fainting or losing control. Clark (1996) points out that when defined in this way, occasional panic attacks are common in all anxiety disorders. However, the diagnosis of panic disorder refers to individuals who experience recurrent panic attacks, at least some of which come on unexpectedly. To be diagnosed with panic disorder, individuals should additionally have ongoing concerns about the possibility of having further panic attacks, be worried about the consequences of panicking or make significant changes to their lifestyles as a result of their worries about having a panic attack.

A diagnosis of panic disorder with agoraphobia (PD-A) is given to those who avoid 'unsafe' places or situations in which they think that panic attacks are likely to occur or from which a rapid escape might be difficult. Such situations include being in supermarkets, theatres and cinemas, travelling in aeroplanes or in underground trains, driving unaccompanied and so on.

\section{The Cognitive Theory of Panic}

Falling, as it does, under the umbrella of the cognitive-behavioural therapeutic tradition (CBT), Rational Emotive Behaviour Therapy (REBT) bases its conceptualisation of panic disorder with or without agoraphobia (PD-A) in large part on the cognitive-behavioural conceptualisation of PD-A.

The current dominant model for understanding the aetiology of panic attacks and Panic Disorder is the cognitive model developed by Clark (1986). According to Clark, "the critical event [in the panic sequence] is the misinterpretation of certain bodily sensations... The sensations are often a consequence of... preceding anxiety, which in turn is due to anticipating an attack or some other anxiety-provoking event unrelated to panic." (Clark 1996, p. 320).

According to Clark's (1986) model, individuals who develop panic disorder after an initial attack do so because they develop an enduring tendency to interpret certain bodily sensations in a catastrophic manner. The individual then becomes hypervigilant for cues that another attack may be about to occur. These cues are formed of sensations that the individual has come to associate with panic, and may, as discussed by Clark (1986, pp. 320-322) arise from "normal" activities such as physical exercise or as a result of the arousal of the fight-or-flight anxiety response. 


\section{REBT Treatment of Panic Disorder}

Whilst the REBT approach to treating panic disorder is based on Clark's cognitive model of panic, there are certain key features of the REBT view of psychological disturbance which distinguish this modality from general cognitive behaviour therapy (Ellis 1994). These principal features will be outlined briefly before going on to examine the four components of the REBT treatment approach used in this study (assessment, re-education, cognitive restructuring, exposure-in vivo and interoceptive).

REBT, like other cognitive-behavioural approaches to psychotherapy regards emotional disturbance as the product, by and large, of thoughts, beliefs and attitudes that the individual holds about whatever adversity the individual encounters. As Dryden (2002, p. 5) states, "In REBT, beliefs are fully and explicitly evaluative and are at the core of a person's emotions and significant behaviours." Such beliefs are considered to be rational or irrational, according to the four criteria of whether they are: (1) realistic, (2) logical, (3) conducive to helping the individual attain his or her goals, and (4) flexible or non-extreme. Beliefs that are unrealistic, illogical, unhelpful and dogmatic or extreme are deemed to be irrational and are the REBT therapist's principal target for change when attempting to help an individual to experience more healthy emotional states and encourage more healthy behaviours.

Ellis (1977, 1994) and Campbell (1985) (in Bond and Dryden 2000) state that as well as the inflexible or demanding nature of the belief, irrational beliefs contain a secondary, evaluative element that "conveys a personally meaningful content or theme." For example, if the primary irrational belief (or "demand" in REBT terminology) is 'I must control my anxiety,' then the secondary belief would consist of an "awfulising" belief such as, 'It would be awful (i.e., 100\% bad) not having control over my anxiety,' and/or a "low frustration tolerance" belief such as, 'Not having such control would be intolerable,' and/or a self-depreciation belief such as, 'Not having such control means I am a weak person.' Panic disorder, when conceptualised according to the REBT view of emotional disturbance, results not just from misinterpreting somatic symptoms and then catastrophising about the symptoms, but from holding 'demanding', 'awfulising' and 'low frustration tolerance' (LFT) beliefs about the consequences of the symptoms. It is the focus on disputing these irrational beliefs that differentiates REBT from other cognitivebehavioural therapies.

In the absence of a standardised REBT manual, the approach outlined by Yankura (1997) in his book chapter forms the basis of the REBT treatment protocol used in this study. Yankura's approach incorporates the following five major features:

- Re-education about panic attacks

- Breathing training and relaxation training

- Cognitive restructuring

- Exposure to interoceptive somatic cues

- Exposure to avoided situations and places 
The REBT treatment approach employed in this study differs from Yankura's (1997) protocol in two major respects. First, this experimental protocol comprises only four treatment sessions whereas Yankura's is not time-limited (and the example given in his book chapter spans 15 sessions), and, second, breathing retraining and relaxation training do not form an integral part of the experimental protocol whereas they do for Yankura. The reasons for these divergences from Yankura's protocol are detailed below.

Limiting the number of sessions to a contract of four sessions, follows evidence from work by Westling and Öst (1999) that brief interventions have a degree of efficacy. Westling and Ost carried out a pilot study into the use of four sessions of general CBT, each of 1 hour duration, with statistically significant reductions at follow-up on measures of panic attacks, agoraphobia, general anxiety and depression.

With regard to breathing and relaxation training, although most self-help texts and practitioner manuals available in the UK as well as in the United States promote the practice of these techniques (e.g., Rachman 2004; Silove and Manicavasagar 1997; Westbrook and Rouf 1998; Tricket 1992), the influential Oxford Group led by David M Clark and Paul Salkovskis dropped the use of these techniques from their treatment programmes in the mid-1990s (Salkovskis 2007). Despite equivocal findings for breathing retraining (Meuret 2003) the Oxford Group did so for much the same the reasons as those cautioned by Yankura (1997); that concentrating on breathing control or relaxation might prevent individuals from developing a more helpful and rational way of thinking about their attacks. More specifically, individuals might prevent themselves from learning that a panic attack might not occur even in situations where one was expected. Furthermore, these techniques foster an idea that controlled breathing can eliminate panic attacks leading in turn to obsessive self-monitoring of the individual's breathing and/or an inappropriate use of breathing techniques as a safety-seeking behaviour.

There are four distinct stages to the REBT treatment employed in this study: assessment, re-education about panic (psychoeducation), cognitive restructuring, and exposure-both interoceptive and in vivo. These are outlined as follows:

\section{Assessment}

In common with other CBT approaches, the first stage in treating PD-A is the assessment phase, the purpose of which is to gather information from the individual about the irrational beliefs which will then be targeted for change during the cognitive restructuring stage later in therapy. By questioning the individual about their experiences of panic-most usually, their most recent panic attack, a typical panic attack and their first panic attack-a model can be created of their idiosyncratic panic cycle. This model would include the sensations they experience, the inferences they reach about the sensations and the irrational beliefs that are triggered by the inferences, along with the safety-seeking behaviours they might engage in and attempts they might make to avoid panicking. This conceptualisation is shared with the individual and corrected if they believe it is inaccurate. 


\section{Psychoeducation}

The second stage in the REBT treatment of PD-A is re-education or "psychoeducation". This entails teaching the individual the details of the "fight or flight" response (Cannon 1927), including the physiological and psychological symptoms associated with the fight-or-flight response, and then conceptualising a number of the individual's panic episodes according to the REBT model of panic (see Fig. 1 for a representative conceptualisation). This conceptualisation is shared with the individual and updated as necessary throughout therapy.

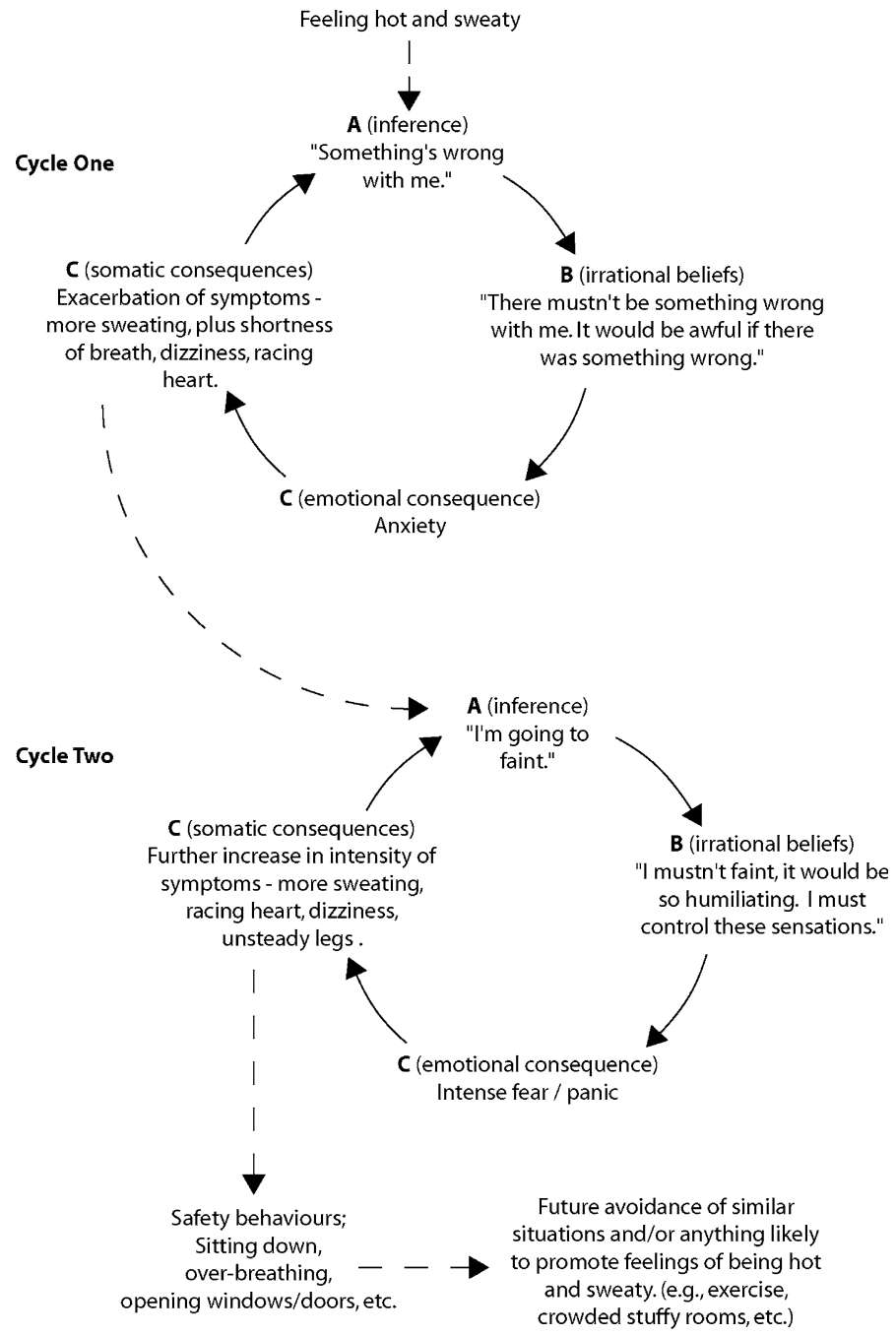

Fig. 1 REBT conceptualisation of an idiosyncratic panic cycle 


\section{Cognitive restructuring}

The third step in the REBT treatment programme is cognitive restructuring. This involves the identification of the irrational beliefs about the panic sensations and about the consequences of panic (e.g., I will faint, and that will be "awful"), and the disputing of these beliefs. By using standard REBT disputing techniques (e.g., Dryden 2002) panic sufferers are helped to understand in empirical, logical and pragmatic terms; (a) why they do not have to control the sensations, (b) why not being able to control the sensations is not awful and, (c) why they can tolerate not controlling the sensations. The rational alternatives of; preferring but not demanding to be in control of the sensations, of viewing this as bad but not awful and of it being unpleasant but not intolerable, are reinforced with cognitive, emotive and behavioural techniques such as role-reversal, disputing, rational portfolios, zigzag forms, and so on (e.g., Dryden 1995).

\section{Exposure}

The important next step in treatment is exposure, both in the clinical setting (interoceptive) and in vivo. Exposure exercises serve to build upon the individual's cognitive disconfirmation of their irrational beliefs about panic symptoms and to reinforce their conviction in their rational beliefs. Also referred to as "exposure and response-prevention" (e.g., Wells 1997), particularly in relation to obsessivecompulsive disorder, in vivo exposure is only fully effective when the individual both enters the feared situation and drops any covert avoidance or safety-seeking behaviours-for example distraction, breathing control, and so on (Neenan and Dryden 2000).

\section{Visual-Kinaesthetic Dissociation}

Visual-kinaesthetic dissociation is, according to Dietrich (2000) "an exposure-based approach that helps individuals attain a degree of kinesthetic [sic] detachment from kinesthetic memories of trauma [or in the case of this study, a panic attack episode] and thereby enables them to process the event(s) from a de-centred perspective. Individuals basically attain a 'visual-kinesthetic reframe' of the experience." The technique was developed by the originators of Neuro-Linguistic Programming (NLP), Bandler and Grinder (1979) based on work originally by Erich Fromm who described the process as a method for dissociating the 'observing ego' from the ‘experiencing ego' (Dietrich 2000).

There are a number of ways of delivering VKD, all involve primary dissociation from the traumatic memory, such as imagining watching the event on a television or cinema screen, but some include a second level of dissociation, and others involve the individual 'entering into' the events on the screen and effectively reliving the remembered event whilst retaining control over the way the events occur and conclude (Weitzenhoffer 2000). This process bears remarkable resemblance to a CBT image-modification technique in which patients are encouraged to hold a negative image in their mind and "then run it on until they see the positive 
resolution," (Clark 1999, p. S18). Similarities also exist with imagery rescripting techniques described by Holmes and others (e.g., Grey et al. 2002; Day et al. 2004; Holmes et al. 2007; Hirsch and Holmes 2007) in which intrusive images are traced back to the original traumatic experience and then reappraised or "cognitively restructured' in light of current experience. The major difference between the CBT image-modification techniques and VKD is that in VKD no attempt is made to examine the meaning the individual attaches to the image, nor to overtly restructure the cognitions associated with the content of the traumatic imagery-these two features being central to CBT imagery-rescripting.

The process of VKD takes place with the individual in a state of relaxationusually induced by using progressive muscle relaxation (PMR). This technique is familiar to many REBT/CBT therapists as a method of helping individuals who experience feelings of anxiety to achieve a lower level of general arousal. PMR is also one of the principal methods employed by hypnotherapists to induce a hypnotic trance state. Once the individual is in a relaxed state or "relaxation trance", VKD follows a number of discrete steps. The first step is to ask the individual to imagine being in a peaceful and relaxing safe place. The individual is also asked to imagine that in their 'safe' or 'special' place there is a television or cinema screen with a video player and remote control facility that they are able to control. The participant is then told that a film sequence of one of their panic attack episodes will play out on the screen in front of them, starting at a point before they began to feel panicky through to a point in time when they began to feel calm once more.

The next step is to have the person imagine floating out of their body to a vantage point from where they can see themselves watching the screen, but cannot actually see the picture itself. The individual is then instructed to let the film sequence play out on the screen in fast-forward motion, whilst they observe the image of themself calmly watching the picture on the screen. When the sequence is finished they are instructed to float back into themself in front of the screen and to watch (and hear) the sequence on the screen as it rewinds at high speed back to the beginning. The individual can then play the sequence forward in fast motion and is instructed to watch the moving image on the screen play through from the beginning of the sequence to the end. At the end of the sequence they are then instructed to float into the image of themself on the screen in the video/film sequence and to experience at first hand (i.e., associated rather than dissociated from the experience) what it feels like to be rewinding back through the experience at high speed. Once they have rewound the sequence back to the beginning the individual is instructed to drift back out of the screen and to 'relax' in their 'special' or 'safe' place and to once again watch the film sequence play forward in fast motion. This process of 'associating' into the sequence and experiencing the sequence occur in reverse at high speed, followed by 'dissociating' from the experience and watching it play forward is carried out several times before the individual is finally asked to watch a new film or video clip of themself reacting calmly in a future situation in which they might previously have panicked, and to drift into themself in the film and to be aware of how it feels to have a sense of calmness in such a situation.

This final step is similar to the REBT practice of imaginal rehearsal of noxious situations, referred to as rational-emotive imagery (Walen et al. 1992), although in 
the case of VKD this occurs without the REBT practice of mental repetition of rational beliefs and rational coping statements. There are also similarities to an image-modification process described by Wells (1997, p. 156) in relation to hypochondriasis or health anxiety. In this disorder, which is not unrelated to panic disorder, featuring as it does distorted cognitions about somatic sensations, Wells argues that individuals typically block or suppress mental images of health-related problems before they reach their worst point. He discusses using imagery to enable individuals to not only critically evaluate salient points of their fear-related beliefs but also to engage in corrective imagery: modifying the ending of their imagined sequence of events, and letting the images run to a new conclusion. Applying this theory to panic disorder, it may be that patients re-traumatise themselves by holding images of the full negative affective state of panic without running the image through to the point after the event at which their arousal level diminishes. It is this process that is facilitated in the VKD procedure.

There are three discrete phases of the VKD condition in this study, assessment, psychoeducation and visual-kinaesthetic reframing. As with the REBT protocol, the first stage in the VKD process is assessment of the individual's idiosyncratic pattern of panic. The pattern of assessment is the same as that for REBT: building a conceptualisation of the individual's idiosyncratic panic cycle based on the REBT model, which is shared with and if necessary modified by the individual.

Assessment is followed by the second stage, psychoeducation. In the VKD condition the psychoeducation component comprises an explanation of the fight-orflight response, the generation of the individual's panic cycle and identification of the irrational beliefs (demands, awfulising, low-frustration tolerance). There is no attempt to dispute or question the validity of the irrational beliefs.

The third and final stage is the visual-kinaesthetic reframing of the Panic Attack episode.

\section{Previous Experiments}

Empirical support for the therapeutic interventions used in both arms of this study is weak. NLP techniques are not known to be supported by a great deal of, if any, true experiments, but it is more surprising to find the use of Rational Emotive Behaviour Therapy for panic disorder has been subjected to little scientific testing. A search of electronic databases, PubMed, MedLine, PsycINFO, and PsycARTICLE, identified only three journal articles relating to the use of REBT with panic disorder. However, on examination of the texts, none of these articles describes a true experimental design and in fact none features REBT as a treatment for panic disorder.

Albert Ellis (2001), the originator of REBT, states that cognitive-behavioural therapies have a large body of scientific research data in support of the various treatment strategies employed. This may be true in respect of general cognitive behaviour therapy for panic disorder (Taylor 2000) but it is not the case as far as REBT is concerned. Empirical support for VKD is as weak as that for REBT, and not just in relation to panic disorder. A search of electronic databases, PubMed, MedLine, PsycINFO, and PsycARTICLE, found no references for 'panic' as a search term when combined with either, 'visual kinaesthetic dissociation', 'VKD', 
'V-KD', or 'V/KD'. Only one reference was retrieved using any of these terms alone-i.e., without the additional criterion of the word, 'panic'. The retrieved text, by Dietrich (2000) is a literature review of VKD. Examination of the bibliography in Dietrich's text led to the retrieval of three further research articles, all of which were single-group case studies researching the use of VKD for post-traumatic stress disorder. The VKD procedure is sometimes referred to as the 'Fast Phobia technique" or "Fast Phobia Rewind" by hypnotherapists. A search of the term "fast phobia" found one hit for a text by Guy and Guy (2003); a multiple case-study nonexperimental design of 30 individuals suffering from PTSD or "partial PTSD" published in a non-peer reviewed journal. Despite the drawbacks of this report having no control or comparison group and the difficulties of generalising the results to guide clinical practice, the findings that Guy \& Guy report are encouraging. They state that none of the 30 participants rated the VKD intervention as a failure. $40 \%$ rated VKD as "extremely successful", 53\% as "successful", and 7\% as "acceptable". Measured on an unspecified wellbeing scale the mean score prior to the intervention was 12 out of 50 and, 7 to 10-days after the treatment, had risen to 30.3 out of 50. At six-month follow-up the rating had further increased to 32.2 out of 50. To achieve this effect Guy and Guy state that 17 participants needed only one session of VKD, 11 needed two and two participants needed three session. They say that in no case did the same trauma need to be treated twice and, in cases where the participant had experienced a single trauma only one session was needed.

In the present study, it was hypothesised that both conditions, REBT and VKD, would show significant improvement on all scores for panic. There was no clear prediction that either condition would be superior to the other, nor if either condition would have a significant effect on scores of depression or anxiety.

\section{Method}

\section{Participants}

Participants were 6 men (33.3\%) and 12 women (66.6\%) meeting DSM-IV (APA 1994) criteria for panic disorder with or without agoraphobia, who responded to media advertisements offering four sessions of free CBT treatment for panic disorder as part of a research trial.

Forty-three individuals responded by telephone to the published editorial features. All were screened for inclusion in the study by asking them to complete, over the telephone, a questionnaire (Panic Disorder Questionnaire, PDQ) based on the section in the Anxiety Disorders Interview Schedule (ADIS-IV; Brown et al. 1994) which covers panic disorder. Adaptations made by the first author consisted of additional questions to limit confounding variables such as: the prior and current use of prescribed and non-prescribed medications, engagement in other therapies (whether orthodox or alternative), co-morbid psychiatric conditions and physical illnesses, and ailments that might mimic panic symptoms.

Thus, respondents to the advertisements were excluded if they: (a) had not experienced panic attacks in the previous month, (b) were engaged in variable 
medication regimens, (c) were receiving another form of therapy (for any condition, not confined solely to PD-A), (d) had been engaged in either CBT/REBT or hypnotherapy during the previous 12 months, (e) demonstrated evidence of an organic mental disorder, schizophrenia, alcohol or drug dependence, cardiovascular disease, asthma, epilepsy, thyroid disorders, (f) were pregnant, (g) were suffering clinically significant co-morbid depression and $(\mathrm{h})$ were experiencing panics more appropriately ascribed to a specific phobia, PTSD, or OCD.

\section{Participant Characteristics}

Of the 43 respondents to the advertisements, 22 individuals were considered suitable and were entered into the study. Four of these 22 were subsequently excluded for changing medication during the study or for disclosing pre-existing medical problems. These four continued to receive the treatments but their data were not entered in the study. The 18 participants who completed the trial ranged in age from 23 to 65 years with a mean age of $46(\mathrm{SD}=13$ years), and had experienced panic attacks for between two months and 30 years with a mean duration of 9.52 years $(\mathrm{SD}=8.98$ years).

These data along with the previously mentioned proportion of $66.6 \%$ female participants in the sample, correspond with the data from most studies into PD-A, in which approximately two-thirds of participants who have panic disorder with agoraphobia are female. The age range of the sample also fits the general PD-A demography in that it is relatively rare for the onset of panic disorder to occur before the age of 15 , with the disorder being roughly twice as frequent in the 25 to 44 -year age range as it is in the 45 to 64-year-old group (Rachman 2004).

\section{Assignment to Groups}

Participants were randomly allocated to each condition inasmuch as they were assigned to the conditions alternately when each participant's baseline questionnaires were received in the post by the researcher.

\section{Design}

The study is a two-way between-groups pre-test/post-test experimental design with baseline and follow-up measures. Owing to time restrictions, it was anticipated that the study would recruit limited participants, recruitment having to be halted at a point that allowed all participants sufficient time to complete the pre-test/post-test measures. Power requirements were therefore not calculated. The decision to restrict the number of groups was taken in order that each group might have the largest number of participants possible. In compensation for the absence of a control group a baseline measure was used.

\section{Instruments/Measures}

Initial screening to gauge suitability of participants for the research experiment was conducted using the panic disorder section of the Anxiety Disorders Interview 
Schedule (ADIS-IV) (Brown et al. 1994) as above. Once accepted onto the trial and to obtain baseline, pre-test/post-test and follow-up measures, participants completed four self-report questionnaires; the Agoraphobic Cognitions Questionnaire (ACQ; Chambless et al. 1984), the Panic Attack Symptoms Questionnaire (PASQ; Clum et al. 1990), the Hospital Anxiety and Depression Scale (HADS; Zigmond and Snaith 1983), and the Global Panic Rating (GPR). The GPR is an instrument developed by the first author for this study and comprises three items each rated on a Likert scale from 0 to 8 , thus giving a total range of 0 to 24 . The items score on the three main criteria for diagnosis of panic disorder; fear of panic, fear of the consequences of panic and, debilitating effects of the fear of panic. As a novel instrument there are no data for internal consistency, or stability.

Therapist

Both treatment conditions in this study were delivered solely by the first author who is a trained hypnotherapist, registered with the United Kingdom Council for Psychotherapists (UKCP) as a Hypno-psychotherapist, and at the time of the study was studying for a Master of Science Degree in Rational Emotive Behaviour Therapy at Goldsmiths University of London run by the second author.

\section{Procedure}

Participants who responded by telephone to the recruitment adverts were assessed by telephone and, if meeting the research trial criteria, were then sent an information sheet, a consent form and a set of the four questionnaires (ACQ, PASQ, HADS, GPR). When individual participants' questionnaires were received by the first author, the participant was allocated to a treatment condition, each treatment being assigned on an alternating basis. Thus, the first participant who returned the questionnaires was assigned to the REBT condition, the second to the VKD condition, the third to REBT, the fourth to VKD, and so on. The first session for all participants took place a minimum of 4 weeks after receipt of the questionnaires and allocation to groups. At the beginning of the first session, an overview of the treatment plan for the four sessions was explained to participants and they were then re-tested on the questionnaires before the treatment element of the first session began. The three subsequent sessions for both groups took place at weekly intervals thereafter. At the end of the fourth session participants were again re-tested on the questionnaires. Four weeks after the fourth and final session for each participant, they were again sent the questionnaires by post with a stamped addressed envelope for their return. Data were then entered into an SPSS spreadsheet for statistical analysis.

\section{Results}

Following random allocation to treatment condition groups the demographic data for the two groups were analysed using a one-way analysis of variance (ANOVA) 
for independent samples, with an alpha level of .05. The results were not statistically significant for any of the demographic variables (age, sex, ethnicity, education, marital status, number of years of PD-A), indicating that the two groups did not differ before receiving the treatment interventions.

Using an alpha level of .05 , data for the within-subjects effects of time were analysed using a repeated-measures analysis of variance (ANOVA), time and condition being the independent variables. The results are given in Table 1.

The results of this multivariate analysis showed that there was a significant main effect of time pre-test/post-test; $F(15,138)=4.78, p<.001$, and a significant interaction effect of time and condition $F(15,138)=2.20, p<.009$. Following the multivariate test, separate univariate ANOVA tests were run for each of the five dependent variables: HADS D (depression), HADS A (anxiety), ACQ, PASQ, and GPR. The univariate tests found highly significant results for each dependent variable across both treatment conditions: these results were; depression, $F(3,48)=22.38, p<.001 ;$ anxiety, $F(3,48)=36.52, p<.001 ; \mathrm{ACQ}, F(3,48)=$ $45.19, p<.001 ;$ PASQ, $F(3,48)=47.93, p<.001 ;$ GPR, $F(3,48)=162.02$, $p<.001$. These results are represented in Table 2 .

A univariate ANOVA test of between-subjects effects, averaged across time, was not statistically significant at $p<.05$, indicating that there were no significant differences between the two groups (see Table 3, below). The scores for each dependent variable were: depression, $F(1,16)=.106$, NS; anxiety, $F(1,16)=.003$, NS; ACQ, $F(1,16)=.374$, NS; PASQ, $F(1,16)=.659$, NS; GPR, $F(1,16)=$ .3.586, NS.

The univariate tests detailed in Table 2 show that the interaction effect discovered in the multivariate ANOVA is the result of a significant interaction effect of time and condition for two dependent variables, HADS-A (anxiety) $[F(3,48)=4.51, p<.007]$ and $\operatorname{GPR}[F(3,48)=5.16, p<.004]$. The other dependent variables, HADS-D (depression), ACQ, and PASQ were not significant, although at $F(3,48)=2.32, p<.088$, ACQ was marginally significant. The data for the interaction effects as detailed in Table 4 are also represented in graph form in Fig. 2, and show a greater change in scores from baseline to follow-up for the VKD group.

Based on the interaction effects shown in these results, separate regression analyses were carried out to assess which predictor variables accounted for the greater improvement in the VKD group. However, the sample size was too small for the inferential statistics to be meaningful and therefore the results are not reported here.

Table 1 Multivariate test of within-subjects effects of time

\begin{tabular}{lccccr}
\hline Within subjects effect & Value & $F$ & Hypothesis $d f$ & Error $d f$ & Sig. \\
\hline Time & 1.02 & 4.78 & 15.00 & 138.00 & .000 \\
Time x Condition & .58 & 2.20 & 15.00 & 138.00 & .009 \\
\hline
\end{tabular}

Tests are based on averaged treatment variables 
Table 2 Univariate test of within-subjects effects of time

\begin{tabular}{lllllrr}
\hline & Measure & Sum of squares & $d f$ & Mean square & $F$ & Sig. \\
\hline Time & HADS depression & 319.04 & 3 & 106.35 & 22.38 & .000 \\
& HADS anxiety & 741.83 & 3 & 247.28 & 36.52 & .000 \\
& ACQ & 11.62 & 3 & 3.87 & 45.19 & .000 \\
& PASQ & $24,386.44$ & 3 & $8,128.81$ & 47.93 & .000 \\
& GPR & $2,277.26$ & 3 & 759.09 & 162.02 & .000 \\
Time $\times$ Condition & HADS depression & 9.60 & 3 & 3.19 & .67 & .573 \\
& HADS anxiety & 91.61 & 3 & 30.54 & 4.51 & .007 \\
& ACQ & .59 & 3 & .19 & 2.32 & .088 \\
& PASQ & 784.44 & 3 & 261.48 & 1.54 & .216 \\
& GPR & 72.54 & 3 & 24.18 & 5.16 & .004 \\
& HADS depression & 228.11 & 48 & 4.75 & & \\
& HADS anxiety & 325.06 & 48 & 6.77 & & \\
& ACQ & 4.11 & 48 & .086 & & \\
& PASQ & $8,140.11$ & 48 & 169.59 & & \\
& GPR & 224.89 & 48 & 4.68 & &
\end{tabular}

Table 3 Univariate test of between-subjects effects averaged across time

\begin{tabular}{lllllll}
\hline Source & Measure & Sum of squares & $d f$ & Mean square & $F$ & Sig. \\
\hline Intercept & HADS depression & $3,712.35$ & 1 & $3,712.35$ & 125.63 & .000 \\
& HADS anxiety & $8,756.06$ & 1 & $8,756.06$ & 410.37 & .000 \\
& ACQ & 260.72 & 1 & 260.72 & 351.76 & .000 \\
& PASQ & $166,656.89$ & 1 & $166,656.89$ & 87.92 & .000 \\
& GPR & $8,504.25$ & 1 & $8,504.25$ & 322.61 & .000 \\
\multirow{5}{*}{ Condition } & HADS depression & 3.12 & 1 & 3.12 & .106 & .749 \\
& HADS anxiety & .06 & 1 & .06 & .003 & .960 \\
& ACQ & .28 & 1 & .28 & .374 & .549 \\
& PASQ & $1,250.00$ & 1 & $1,250.00$ & .659 & .429 \\
& GPR & 94.53 & 1 & 94.53 & 3.586 & .076 \\
& HADS depression & 472.78 & 16 & 29.55 & & \\
& HADS anxiety & 341.39 & 16 & 21.34 & & \\
& ACQ & 11.86 & 16 & .74 & & \\
& PASQ & $30,330.11$ & 16 & $1,895.63$ & & \\
& GPR & 421.78 & 16 & 26.36 & &
\end{tabular}

\section{Discussion}

This study sought to experimentally test two treatment methods, Rational Emotive Behaviour Therapy (REBT) and Visual/Kinaesthetic Dissociation (VKD), in the treatment of Panic Disorder with or without Agoraphobia (PD-A). 
Table 4 Treatment independent variable $\times$ time

\begin{tabular}{|c|c|c|c|c|c|c|}
\hline \multirow[t]{2}{*}{ Measure } & \multirow{2}{*}{$\begin{array}{l}\text { Treatment } \\
\text { independent } \\
\text { variable }\end{array}$} & \multirow[t]{2}{*}{ Time } & \multirow[t]{2}{*}{ Mean } & \multirow[t]{2}{*}{ Std. Error } & \multicolumn{2}{|c|}{$95 \%$ confidence interval } \\
\hline & & & & & Lower bound & Upper bound \\
\hline \multirow[t]{8}{*}{ HADS depression } & \multirow[t]{4}{*}{ REBT } & 1 & 9.22 & 1.06 & 6.98 & 11.47 \\
\hline & & 2 & 8.56 & 1.04 & 6.35 & 10.76 \\
\hline & & 3 & 4.44 & 1.14 & 2.02 & 6.87 \\
\hline & & 4 & 5.67 & 1.16 & 3.20 & 8.13 \\
\hline & \multirow[t]{4}{*}{ VKD } & 1 & 9.67 & 1.06 & 7.42 & 11.91 \\
\hline & & 2 & 9.67 & 1.04 & 7.46 & 11.88 \\
\hline & & 3 & 5.33 & 1.14 & 2.91 & 7.76 \\
\hline & & 4 & 4.89 & 1.16 & 2.42 & 7.36 \\
\hline \multirow[t]{8}{*}{ HADS anxiety } & \multirow[t]{4}{*}{ REBT } & 1 & 14.44 & .95 & 12.44 & 16.45 \\
\hline & & 2 & 12.00 & 1.08 & 9.71 & 14.29 \\
\hline & & 3 & 9.00 & 1.09 & 6.70 & 11.30 \\
\hline & & 4 & 8.56 & 1.18 & 6.06 & 11.05 \\
\hline & \multirow[t]{4}{*}{ VKD } & 1 & 14.89 & .95 & 12.88 & 16.90 \\
\hline & & 2 & 15.56 & 1.08 & 13.26 & 17.85 \\
\hline & & 3 & 6.67 & 1.09 & 4.36 & 8.97 \\
\hline & & 4 & 7.11 & 1.18 & 4.62 & 9.60 \\
\hline \multirow[t]{8}{*}{ ACQ } & \multirow[t]{4}{*}{ REBT } & 1 & 2.25 & .20 & 1.83 & 2.66 \\
\hline & & 2 & 2.05 & .18 & 1.67 & 2.43 \\
\hline & & 3 & 1.48 & .15 & 1.17 & 1.79 \\
\hline & & 4 & 1.59 & .14 & 1.29 & 1.89 \\
\hline & \multirow[t]{4}{*}{ VKD } & 1 & 2.53 & .20 & 2.12 & 2.95 \\
\hline & & 2 & 2.37 & .18 & 1.99 & 2.75 \\
\hline & & 3 & 1.48 & .16 & 1.17 & 1.79 \\
\hline & & 4 & 1.48 & .14 & 1.18 & 1.78 \\
\hline \multirow[t]{8}{*}{ PASQ } & \multirow[t]{4}{*}{ REBT } & 1 & 61.44 & 7.74 & 45.04 & 77.85 \\
\hline & & 2 & 57.11 & 9.33 & 37.34 & 76.89 \\
\hline & & 3 & 30.22 & 7.28 & 14.79 & 45.66 \\
\hline & & 4 & 27.00 & 8.20 & 9.62 & 44.39 \\
\hline & \multirow[t]{4}{*}{ VKD } & 1 & 72.67 & 7.74 & 56.26 & 89.07 \\
\hline & & 2 & 74.78 & 9.33 & 55.00 & 94.55 \\
\hline & & 3 & 31.00 & 7.28 & 15.57 & 46.43 \\
\hline & & 4 & 30.67 & 8.20 & 13.28 & 48.05 \\
\hline \multirow[t]{8}{*}{ GPR } & \multirow[t]{4}{*}{ REBT } & 1 & 15.33 & .85 & 13.53 & 17.16 \\
\hline & & 2 & 13.67 & 1.10 & 11.34 & 15.99 \\
\hline & & 3 & 4.67 & 1.14 & 2.26 & 7.08 \\
\hline & & 4 & 5.22 & 1.13 & 2.83 & 7.61 \\
\hline & \multirow[t]{4}{*}{ VKD } & 1 & 18.17 & .85 & 16.37 & 19.97 \\
\hline & & 2 & 18.78 & 1.10 & 16.45 & 21.11 \\
\hline & & 3 & 6.33 & 1.14 & 3.92 & 8.74 \\
\hline & & 4 & 4.78 & 1.13 & 2.39 & 7.17 \\
\hline
\end{tabular}



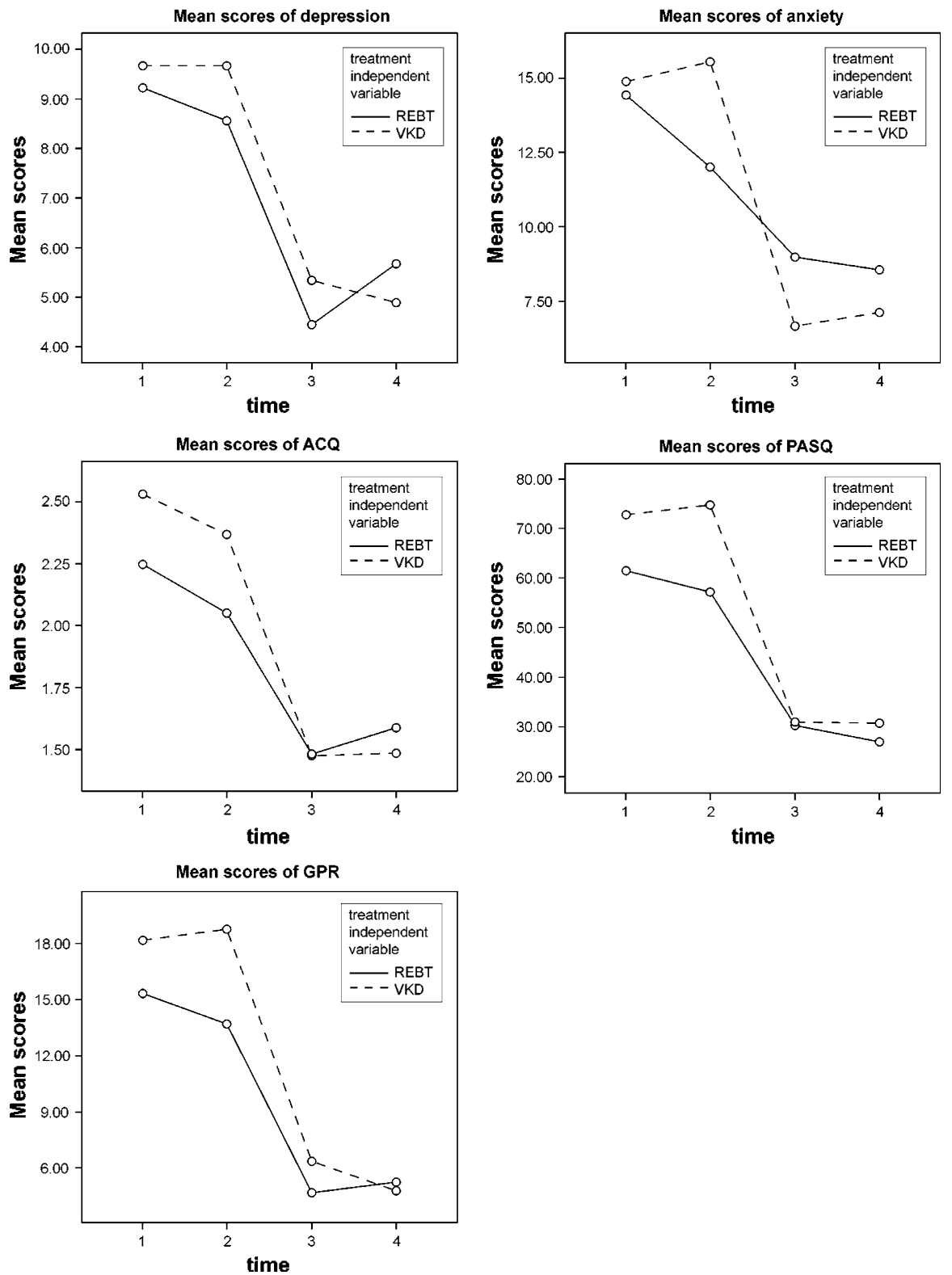

Fig. 2 Interaction effect of treatment condition on measures across time

To date there have been no published experimental tests of the use of REBT for PD-A, and no experimental tests of VKD for any disorder. It would appear that this study is the first to employ the scientific method to test the efficacy of these treatments for PD-A. 
The findings of this study are that four sessions of both treatment conditions brought about highly statistically significant reductions in measures of panic. Both treatment conditions also resulted in highly significant reductions in measures of depression and anxiety.

At four-week follow-up any difference between the groups' scores was nonsignificant, indicating that both treatment conditions were equally efficacious in treating PD-A.

Given that the mean duration of PD-A for the participants prior to treatment was nearly 10 years, and that there was no significant change in the scores during the baseline period it may be concluded with some confidence that the causative factor in the improvement in panic scores was the treatment intervention.

The significant reductions in the ACQ and PASQ scales suggest that by the end of treatment the participants in both groups were thinking less catastrophically and more realistically about previously feared sensations that led to panic attacks and that they were experiencing an absence or shorter duration of the sensations. These reductions in ACQ and PASQ scores were reflected in the Global Panic Rating (GPR) scores. The significant reductions in this measure suggest that, at follow-up, panic was considered less of a problem by the participants than before treatment.

Although depression and anxiety were not targeted directly in either treatment condition, there was a significant reduction in both, as measured by the HADS scale. This is not a surprising finding given the high incidence of co-morbid mood disorders with Panic Disorder (Andrews et al. 2003).

Of note is the significant interaction effect for the VKD group on scores of anxiety (HADS-A) and GPR from pre-treatment to post-treatment. This shows that there was a greater improvement for this group compared to the REBT group. Whilst the between-subjects ANOVA (Table 3) showed no significant differences between the groups, one high scoring participant in the REBT group demonstrated large changes in HADS-A and GPR scores between time points 1 and 2 (baseline and pre-test). The interaction effect on these scores may therefore be an artefact of this participant's scores regressing to the mean. The results of this study highlight the limitations of the small sample size on the generalisability of these data to a wider population of PD-A sufferers, and future studies should employ a larger sample to test if these findings are replicated.

With no REBT or VKD texts available for comparison, these results stand alone in the literature; however they compare favourably with Westling and Öst's (1999) study of four sessions of CBT for panic disorder and with other studies that employed a greater number of sessions of CBT (e.g., Clark et al. 1985). In addition to the positive clinical results of interventions employing only four sessions, the nature of the interventions themselves are of note: REBT, like other cognitivebehavioural psychotherapies, requires extensive training at postgraduate level for therapists to reach a good standard of competence. By contrast, the process of using progressive muscle relaxation to induce a state of relaxation is relatively straightforward and easily learned, as is the VKD intervention itself (which, as the study has shown, can be delivered by reading from a prepared script). Given the less intensive training required to deliver VKD competently this intervention would appear, on the basis of the positive findings of this study, to offer an efficacious 
alternative to more lengthy CBT protocols which necessitate delivery by highly trained clinicians. On the other hand, both treatments were conducted by the first author who, at the time of the study, was a trained hypnotherapist (more relevant to VKD) and had not yet been awarded his Masters degree in REBT. It is thus not possible to make definitive statements concerning the expertise of the therapist and its possible influence on the results of this study. This variable needs to be controlled in future research.

Although the highly statistically significant results of this study for REBT and VKD in relation to panic disorder indicate that both treatment methods are efficacious there are a number of limitations to this study, principally the small sample size and the absence of a long-term follow-up. Notwithstanding the threats to external validity that are inherent in a small sample size the nine participants in each treatment condition in this study were sufficient to allow statistical analyses to reveal meaningful inferences about main and interaction effects. Confidence can therefore be had in the findings of this study. However, the small sample size limits the generalisability of the findings to other populations.

Following this small-scale study a larger scale study is planned which will incorporate:

- A larger sample with a minimum of 20 participants per group, drawn from primary care referrals as well as recruited through media advertisements.

- Delivery of therapy by different therapists, ideally at different locations where the expertise of the therapist is controlled.

- Four groups:

- Wait List (wait time equivalent to 4-week baseline and 4-week treatment)

- REBT

- VKD

- REBT plus VKD.

- The use of an REBT psychoeducation text for the REBT group.

- The use of additional measures, including a measure of irrational beliefs, a more comprehensive measure for co-morbidity, a panic diary to survey the frequency and severity of panics, and participant rating of the credibility of the treatment conditions.

- Tape-recording of sessions to allow rating of adherence, differentiation, purity and quality of treatment by an independent assessor.

- Follow-up measures at no less than 6 months and preferably at 12 months posttreatment.

In summary, given the lack of published data for the two treatment methods tested, this study provides valuable empirical support regarding the efficacy of both treatment methods REBT and VKD, in relation to panic disorder, and indicates that both might be employed to good effect by clinicians. 


\section{References}

American Psychiatric Association. (1994). Diagnostic and statistical manual of mental disorders (4th ed.). Washington, DC: American Psychiatric Association.

Andrews, G., Creamer, M., Crino, R., Hunt, C., Lampe, L., \& Page, A. (2003). The treatment of anxiety disorders. Cambridge: Cambridge University Press.

Bandler, R., \& Grinder, J. (1979). Frogs into princes. London: Eden Grove Editions.

Beamish, P. M., Granello, D. H., \& Belcastro, A. L. (2002). Treatment of panic disorder: Practical guidelines. Journal of Mental Health Counseling, 24(3), 224-246. (p224).

Bond, F. W., \& Dryden, W. (2000). How rational beliefs and irrational beliefs affect people's inferences: An experimental investigation. Behaviour and Cognitive Psychotherapy, 28, 33-43.

Brown, T. A., Di Nardo, P. A., \& Barlow, D. H. (1994). Anxiety disorders interview schedule for DSM-IV. Albany, NY: Graywind Publications.

Campbell, I. (1985). The psychology of homosexuality. In A. Ellis \& M. E. Bernard (Eds.), Clinical applications of rational emotive therapy. New York: Plenum.

Cannon, W. B. (1927). Bodily changes in pain, hunger, fear, and rage. New York: Appleton-CenturyCrofts.

Chambless, D. L., Caputo, C. G., Bright, P., \& Gallagher, R. (1984). Assessment of fear in agoraphobics: The body sensations questionnaire and the agoraphobics questionnaire. Journal of Consulting and Clinical Psychology, 52, 1090-1097.

Clark, D. M. (1986). A cognitive approach to panic. Behaviour Research and Therapy, 24, 461-470.

Clark, D. M. (1996). Panic disorder: From theory to therapy. In P. M. Salkovskis (Ed.), Frontiers of cognitive therapy. New York: Guilford Publications.

Clark, D. M. (1999). Anxiety disorders: Why they persist and how to treat them. Behaviour Research and Therapy, 37, S5-S27.

Clark, D. M., Salkovskis, P. M., \& Chalkey, A. J. (1985). Respiratory control as a treatment for panic attacks. Journal of Behaviour Therapy and Experimental Psychiatry, 16, 23-30.

Clum, G. A., Broyles, S., Borden, J., \& Watkins, P. L. (1990). Validity and reliability of the Panic Attack Symptoms and Cognition Questionnaire. Journal of Psychopathology and Behavioural Assessment, 12, 233-245. Plenum Publishing Crop.

Day, S. J., Homes, E. A., \& Hackmann, A. (2004). Occurrence of imagery and its link with early memories in agoraphobia. Memory, 12(4), 416-427.

Dietrich, A.M. (2000). A review of visual/kinesthetic disassociation in the treatment of posttraumatic disorders: Theory, efficacy and practice recommendations. Traumatology, [Internet]. VI(2), Article 3 (August). Available from http://www.fsu.edu/ trauma/v6i2a3.html. Accessed, 15.01.07.

Dryden, W. (1995). Brief rational emotive behaviour therapy. Chichester: Wiley.

Dryden, W. (2002). Fundamentals of rational emotive behaviour therapy. London: Whurr Publishers.

Ellis, A. (1977). The basic clinical theory of rational-emotive therapy. In A. Ellis \& R. Grieger (Eds.), Handbook of rational-emotive therapy. New York: Springer.

Ellis, A. (1994). Reason and emotion in psychotherapy. Revised and updated. New York: Birch Lane Press.

Ellis, A. (2001). Reasons why rational emotive behaviour therapy is relatively neglected in the professional and scientific literature. Journal of Rational-Emotive \& Cognitive-Behavior Therapy, 19(1), 67-74.

Grey, N., Young, K., \& Holmes, E. (2002). Cognitive restructuring within reliving: A treatment for peritraumatic emotional "hotspots" in posttraumatic stress disorder. Behavioural and Cognitive Psychotherapy, 2002(30), 37-56.

Guy, K., \& Guy, N. (2003). The fast cure for phobia and trauma: Evidence that it works. Human Givens Journal, [Internet]. 9(4). Available from: http://www.humangivens.com/hgi/archive/rewindevi dence.htm. Accessed 15.01.07.

Hirsch, C. R., \& Holmes, E. A. (2007). Mental imagery in anxiety disorders. Psychiatry, 6(4), 161-165.

Holmes, E. A., Arntz, A., \& Smucker, M. R. (2007). Imagery rescripting in cognitive behaviour therapy: Images, treatment techniques and outcomes. Journal of Behavior Therapy and Experimental Psychiatry, 38(4), 297-305.

Meuret, A. E. (2003). Breathing training for treating panic disorder. Behavior Modification, 27(5), 731-754.

Neenan, M., \& Dryden, W. (2000). Essential rational emotive behaviour therapy. London: Whurr. 
Rachman, S. J. (2004). Panic disorder: The facts. Oxford: Oxford University Press.

Salkovskis, P. M. (2007). Cognitive-behavioural treatment for Panic Disorder and Agoraphobia. Centre for Anxiety Disorders \& Trauma, Institute of Psychiatry, Kings College University of London. Unpublished workshop notes [workshop, 23rd and 24th November 2006].

Silove, D., \& Manicavasagar, V. (1997). Overcoming panic. London: Robinson.

Taylor, S. (2000). Understanding and treating panic disorder: Cognitive-behavioural approaches. New York: Wiley.

Tricket, S. (1992). Coping successfully with panic attacks. London: Sheldon Press.

Walen, S. R., DiGiuseppe, R., \& Dryden, W. (1992). A practitioner's guide to rational-emotive behaviour therapy (2nd ed., p. 25). Oxford: Oxford University Press.

Weitzenhoffer, A. M. (2000). The practice of hypnotism (2nd ed.). New York: Wiley.

Wells, A. (1997). Cognitive therapy of anxiety disorders. Chichester: Wiley.

Westbrook, D., \& Rouf, K. (1998). Understanding panic. Oxford: Oxford Cognitive Therapy Centre.

Westling, B. E., \& Öst, L.-G. (1999). Brief cognitive behaviour therapy of panic disorder. Scandinavian Journal of Behaviour Therapy, 28(2), 49-57.

Yankura, J. (1997). REBT and panic disorder with agoraphobia. In J. Yankura \& W. Dryden (Eds.), Using REBT with common psychological problems: A therapist's casebook (pp. 112-157). New York: Springer.

Zigmond, A. S., \& Snaith, R. P. (1983). The hospital anxiety and depression scale. Acta Psychiatrica Scandinavia, 67, 361-370. 